\title{
Elevated intracellular cAMP exacerbates vulnerability to oxidative stress in optic nerve head astrocytes
}

Myoung Sup Shim', Keun-Young Kim², Jung Hyun Bu', Hye Seung Nam', Seung Won Jeong ${ }^{1}$, Tae Lim Park', Mark H. Ellisman², Robert N. Weinreb ${ }^{1}$ and Won-Kyu Ju'

\begin{abstract}
Glaucoma is characterized by a progressive loss of retinal ganglion cells and their axons, but the underlying biological basis for the accompanying neurodegeneration is not known. Accumulating evidence indicates that structural and functional abnormalities of astrocytes within the optic nerve head $(\mathrm{ONH})$ have a role. However, whether the activation of cyclic adenosine $3^{\prime}, 5^{\prime}$-monophosphate (cAMP) signaling pathway is associated with astrocyte dysfunction in the $\mathrm{ONH}$ remains unknown. We report here that the cAMP/protein kinase A (PKA) pathway is critical to ONH astrocyte dysfunction, leading to caspase-3 activation and cell death via the AKT/Bim/Bax signaling pathway. Furthermore, elevated intracellular CAMP exacerbates vulnerability to oxidative stress in ONH astrocytes, and this may contribute to axonal damage in glaucomatous neurodegeneration. Inhibition of intracellular cAMP/PKA signaling activation protects $\mathrm{ONH}$ astrocytes by increasing AKT phosphorylation against oxidative stress. These results strongly indicate that activation of CAMP/PKA pathway has an important role in astrocyte dysfunction, and suggest that modulating CAMP/ PKA pathway has therapeutic potential for glaucomatous $\mathrm{ONH}$ degeneration.
\end{abstract}

\section{Introduction}

Primary open-angle glaucoma (POAG) is characterized by a slow and progressive degeneration of retinal ganglion cells (RGCs) and their axons in the optic nerve $(\mathrm{ON})$, leading to loss of visual function ${ }^{1}$. The factors contributing to axon degeneration in the optic nerve head $(\mathrm{ONH})$ in POAG are not well understood. Accumulating evidence indicates that astrocyte dysfunction accompanied by RGC axon loss is closely associated with the pathogenesis of glaucomatous $\mathrm{ONH}$ degeneration ${ }^{2-4}$. Indeed, structural and functional abnormalities of astrocytes have been reported in the $\mathrm{ONH}$ of experimental glaucoma models as well as patients with $\mathrm{POAG}^{2,3,5,6}$.

\footnotetext{
Correspondence: W-K. Ju (wju@ucsd.edu)

${ }^{1}$ Hamilton Glaucoma Center and Department of Ophthalmology, Shiley Eye Institute, University of California San Diego, La Jolla, CA, USA

${ }^{2}$ Center for Research on Biological Systems, National Center for Microscopy and Imaging Research and Department of Neuroscience, University of

California San Diego, La Jolla, CA, USA

Edited by B. Joseph
}

The ubiquitous second messenger cyclic adenosine $3^{\prime}, 5^{\prime}$ monophosphate (cAMP) in the central nervous system contributes to numerous biological processes including cell growth and death ${ }^{7-10}$. Upon stimulation, cAMP synthesis and its degradation are tightly regulated by adenyl cyclases (ACs) and cyclic nucleotide phosphodiesterases (PDEs), respectively ${ }^{8}$. Previous studies have demonstrated that the basal level of cAMP was significantly higher in the unstimulated glaucomatous $\mathrm{ONH}$ astrocytes from Caucasian American and African American (AA) donors with POAG compared with their unstimulated $\mathrm{ONH}$ astrocytes from normal healthy counterparts ${ }^{11}$. Furthermore, elevated hydrostatic pressure, a mimetic of high intraocular pressure (IOP) in vitro, upregulated the mRNA expression of two transmembrane ACs (tmACs) genes, $A D C Y 3$ (AC3), and $A D C Y 9$ (AC9), in the $\mathrm{ONH}$ astrocytes from AA donors ${ }^{12}$. This suggests an intriguing possibility that the activation of intracellular cAMP signaling pathway may have a role in the pathogenesis of glaucomatous $\mathrm{ONH}$ astrocytes. 
Nevertheless, the precise cellular and molecular mechanism (s) of the activation of cAMP signaling pathway in astrocyte dysfunction in glaucomatous $\mathrm{ONH}$ degeneration remains to be determined. These mechanisms may be related to oxidative stress.

Oxidative stress has long been thought to be an important pathophysiological mechanism in many neurodegenerative diseases including glaucoma ${ }^{2,4,13-15}$. Astrocytes are the responsible cell type for many pathological alterations in the oxidative stress-mediated glaucomatous $\mathrm{ONH}$ degeneration ${ }^{2-4,16}$. Interestingly, activation of the tmACsmediated cAMP/protein kinase A (PKA) signal pathway induced by forskolin is associated with increased vulnerability to hydrogen peroxide $\left(\mathrm{H}_{2} \mathrm{O}_{2}\right)$-induced oxidative stress in rat neocortical astrocytes in vitro ${ }^{17}$. These findings collectively suggest that the elevated intracellular cAMP-mediated PKA signaling pathway contributed to oxidative stress-induced dysfunction of astrocytes during glaucomatous $\mathrm{ONH}$ degeneration.

In the present study, we investigate the impact of activation of cAMP/PKA pathway and its synergistic effect with oxidative stress on $\mathrm{ONH}$ astrocyte dysfunction.

\section{Results}

Elevated cAMP impairs ONH astrocytes, but promotes RGC survival

Intriguingly, our results demonstrated that forskolin treatment significantly increased intracellular cAMP level and PKA activity, accompanying by dose-dependent reduction of cell viability measured by 3-[4, 5-dimethylthiazol-2yl]-2, 5-diphenyl tetrazolium bromide (MTT) assay (Figs. 1a-d). As it has been reported that AC and PDE4 (a cAMP-specific PDE) are two key enzymes that control intracellular cAMP level in $\mathrm{RGCs}^{18}$, we co-treated RGCs with forskolin and rolipram, a specific inhibitor of PDE4, to enhance cAMP-mediated PKA activity and with forskolin and H89 to inhibit cAMP-mediated PKA activity. The elevated cAMP level was positively correlated with viability of RGCs (Fig. 1e), whereas PKA inhibition significantly suppressed cAMP-mediated promotion of cell viability in cultured RGCs (Fig. 1f).

\section{Elevated cAMP inhibits AKT phosphorylation and induces caspase-3-mediated cell death in $\mathrm{ONH}$ astrocytes}

Elevated cAMP significantly decreased the level of AKT phosphorylation (pAKT) at serine 473 (S473) normalized by total AKT in ONH astrocytes (Fig. 2a). Using forskolin together with $\mathrm{H} 89$, a PKA inhibitor, or CE3F4, a specific inhibitor of an exchange protein directly activated by cAMP $(\text { Epac })^{19}$, we further addressed that elevated cAMP-mediated inhibition of AKT phosphorylation was dependent on the PKA pathway but not Epac pathway (Figs. 2b and c). Following prolonged elevation of cAMP levels by forskolin and rolipram $(24 \mathrm{~h})$, the polygonal, fibroblast-like shape of $\mathrm{ONH}$ astrocytes were changed to the extensive stellate-like morphology within a few hours, most likely to be a reactive astrogliosis and this morphological alteration was sustained for $24 \mathrm{~h}$ upon increasing cAMP level (Supplementary Fig. S1). Notably, consistent with compromised cell viability in $\mathrm{ONH}$ astrocytes by the rolipram treatment ( $1 \mathrm{~h}$; Fig. $2 \mathrm{~d}$ ), prolonged elevation of cAMP level not only suppressed AKT phosphorylation but also increased the cleaved caspase-3 protein level in $\mathrm{ONH}$ astrocytes (Figs. 2e and f).

\section{Inhibiting CAMP/PKA pathway prevents Bim/Bax pathway} and caspase-3 activation in $\mathrm{ONH}$ astrocytes

Quantitative real-time reverse transcription (RT)-polymerase chain reaction (PCR) analysis demonstrated that forskolin treatment induced a substantial increase of Bim expression in $\mathrm{ONH}$ astrocytes among apoptosis-related genes that are regulated by NF- $\mathrm{kB}$ (TNF $\alpha, \mathrm{Bcl}-2$, and $\mathrm{Bcl}-$ $\mathrm{xL}$ ) or FoxO (Bim, Bcl-6, Puma, Bnip3, Trail, and FasL) (Fig. 3a). By selecting Bim as an AKT target gene regulated by cAMP, we further observed that PKA inhibition by H89 significantly suppressed Bim mRNA expression in forskolin-treated $\mathrm{ONH}$ astrocytes (Fig. 3b). Insulin-like growth factor 1 (IGF-1) regulates apoptosis and oxidative stress response via phosphatidylinositol-5-bisphosphate 3-kinase (PI3K)/AKT signaling pathway in astrocytes ${ }^{20}$. We found that IGF-1 reversed cell viability that was reduced by forskolin treatment alone in an AKT-FoxOdependent manner in $\mathrm{ONH}$ astrocytes (Fig. 3c and Supplementary Fig. S2). There was no significant difference in lactate dehydrogenase (LDH) release between forskolinand IGF-1-treated ONH astrocytes (Fig. 3d).

Co-immunoprecipitation (Co-IP) revealed that forskolin treatment promoted the interaction between Bim and $\mathrm{Bcl}-$ $\mathrm{xL}$ in $\mathrm{ONH}$ astrocytes (Fig. 3e). In contrast, PKA inhibition blocked this interaction in forskolin-treated $\mathrm{ONH}$ astrocytes (Fig. 3e). Furthermore, prolonged elevation of cAMP levels by forskolin and rolipram $(24 \mathrm{~h})$ significantly increased both Bim and activated Bax protein expression in $\mathrm{ONH}$ astrocytes (Fig. 3f). By co-treatment with forskolin, Rp diastereomers of cAMP (Rp-cAMP), a PKA inhibitor, or H89 in cultured ONH astrocytes for $24 \mathrm{~h}$, inhibition of cAMP/PKA pathway by Rp-cAMP significantly decreased activated Bax and cleaved caspase- 3 protein expression in $\mathrm{ONH}$ astrocytes, whereas the expression level of pAKT S473 in ONH astrocytes was not changed compared with the control treated with forskolin (Fig. 3g). However, interestingly, inhibition of cAMP/PKA pathway by $\mathrm{H} 89$ significantly increased the levels of pAKT S473, whereas cleaved caspase-3 protein expression was not decreased and the expression level of Bax in $\mathrm{ONH}$ astrocytes was not changed (Fig. 3g). In addition, consistent with our result of reduction in activated Bax and caspase-3 protein expression, immunocytochemical 


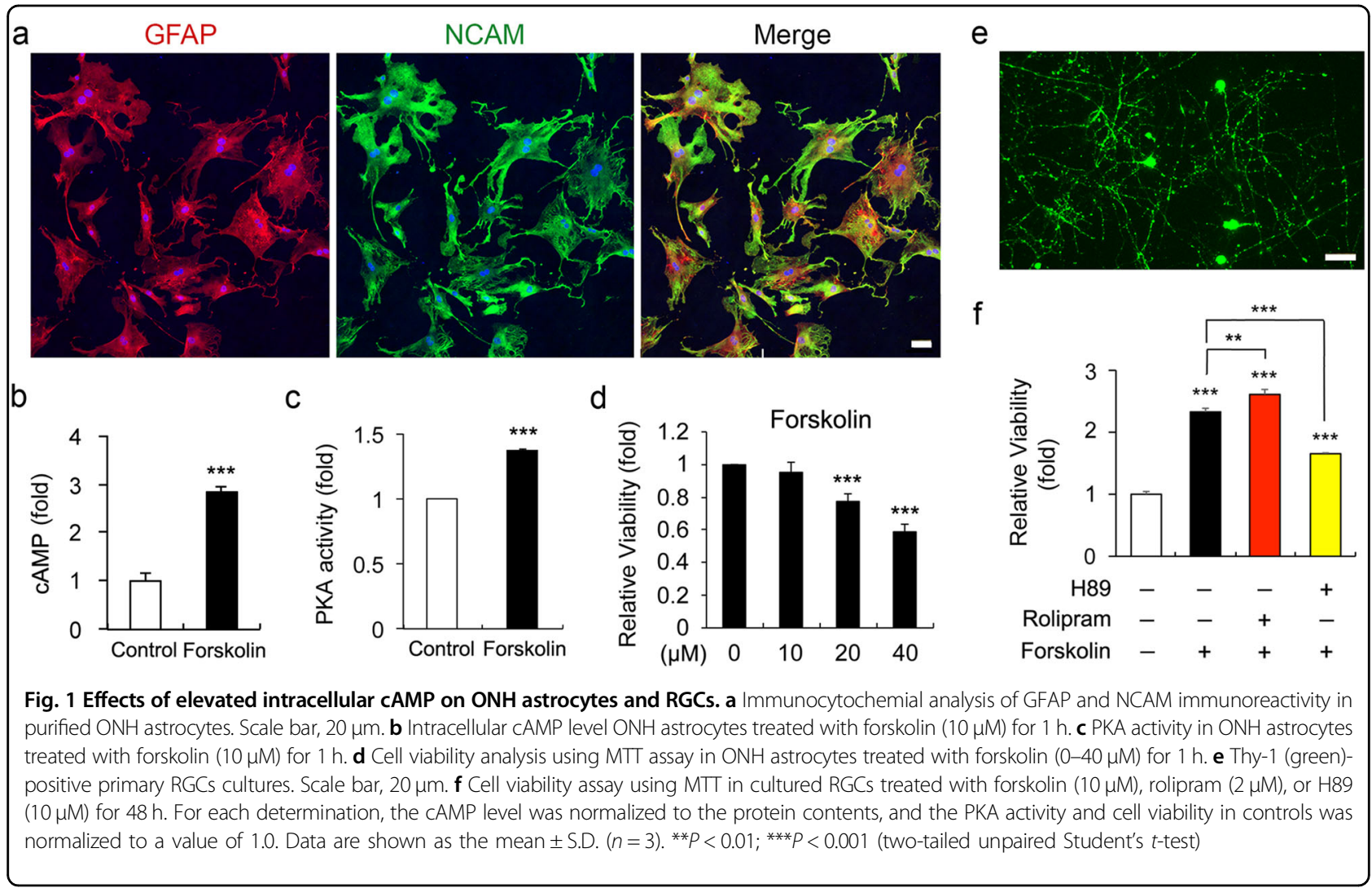

analysis confirmed that inhibition of cAMP/PKA pathway by Rp-cAMP decreased immunoreactivities for activated Bax and caspase-3 protein expression that were increased in forskolin-treated $\mathrm{ONH}$ astrocytes, accompanied by an extensive stellate-like morphology that is likely to be a reactive astrogliosis (Supplementary Fig. S3).

Enhanced cAMP levels are correlated with an increase in activated Bax and caspase-3 expression in glaucomatous ONH astrocytes

Because glaucomatous DBA/2 J mice, an extensively characterized strain that spontaneously develops elevated IOP with aging ${ }^{21}$, show the structural and functional abnormalities of $\mathrm{ONH}$ astrocytes in glaucoma progres$\operatorname{sion}^{3,5,6}$, we used DBA/2 J mice to determine whether glaucomatous $\mathrm{ONH}$ astrocytes alters the expression levels of cAMP, Bax and caspase-3 protein. Serial block-face scanning electron microscopy (SBEM) data sets showed degenerative structural changes in astrocyte processes in the glial lamina of 10-month-old glaucomatous DBA2/J mice, accompanied by axon degeneration (Figs. 4a-c). Further analysis of three-dimensional reconstruction of segmentations of axon bundle and astrocyte processes in SBEM stacks revealed a well-organized longitudinal shape of axon bundle in the glial lamina of DBA/2J-Gpnmb ${ }^{+}$ mice (Fig. 4d), whereas distorted axon bundles were prominent in the glial lamina of glaucomatous DBA/2 J mice (Fig. 4e). On the other hand, astrocytes in the glial lamina of DBA/2J-Gpnmb ${ }^{+}$mice presented an irregular arrangement of processes, which is likely a shape of hook and loop (Fig. $4 \mathrm{f}$ and Supplementary Movie S1). However, although a moderate glaucoma damage caused a loosen arrangement of astrocytic processes (Fig. 4g and Supplementary Movie S2), severe glaucoma damage induced a significant loss of astrocytic processes in the glial lamina of glaucomatous DBA/2 J mice (Fig. 4h and Supplementary Movie S3). Importantly, glaucomatous astrocytes showed an increase of cAMP, Bax, and caspase-3 immunoreactivities (Figs. 5a-c and Supplementary Fig. S4).

\section{Elevated CAMP exacerbates vulnerability to oxidative stress in ONH astrocyte dysfunction}

Of interest, elevated intracellular cAMP by forskolin exacerbated the reduction of cell viability in oxidative stress-induced $\mathrm{ONH}$ astrocytes by a dose-dependent manner (Fig. 6a) and enhanced cAMP level by rolipram significantly decreased cell viability in oxidative stressinduced $\mathrm{ONH}$ astrocytes compared with controls (Fig. 6b). Furthermore, elevated cAMP significantly increased cell death in oxidative stress-induced $\mathrm{ONH}$ astrocytes (Fig. 6c). The splicing of the precursor TNF $\alpha$ mRNA into the mature form is responsive to external 


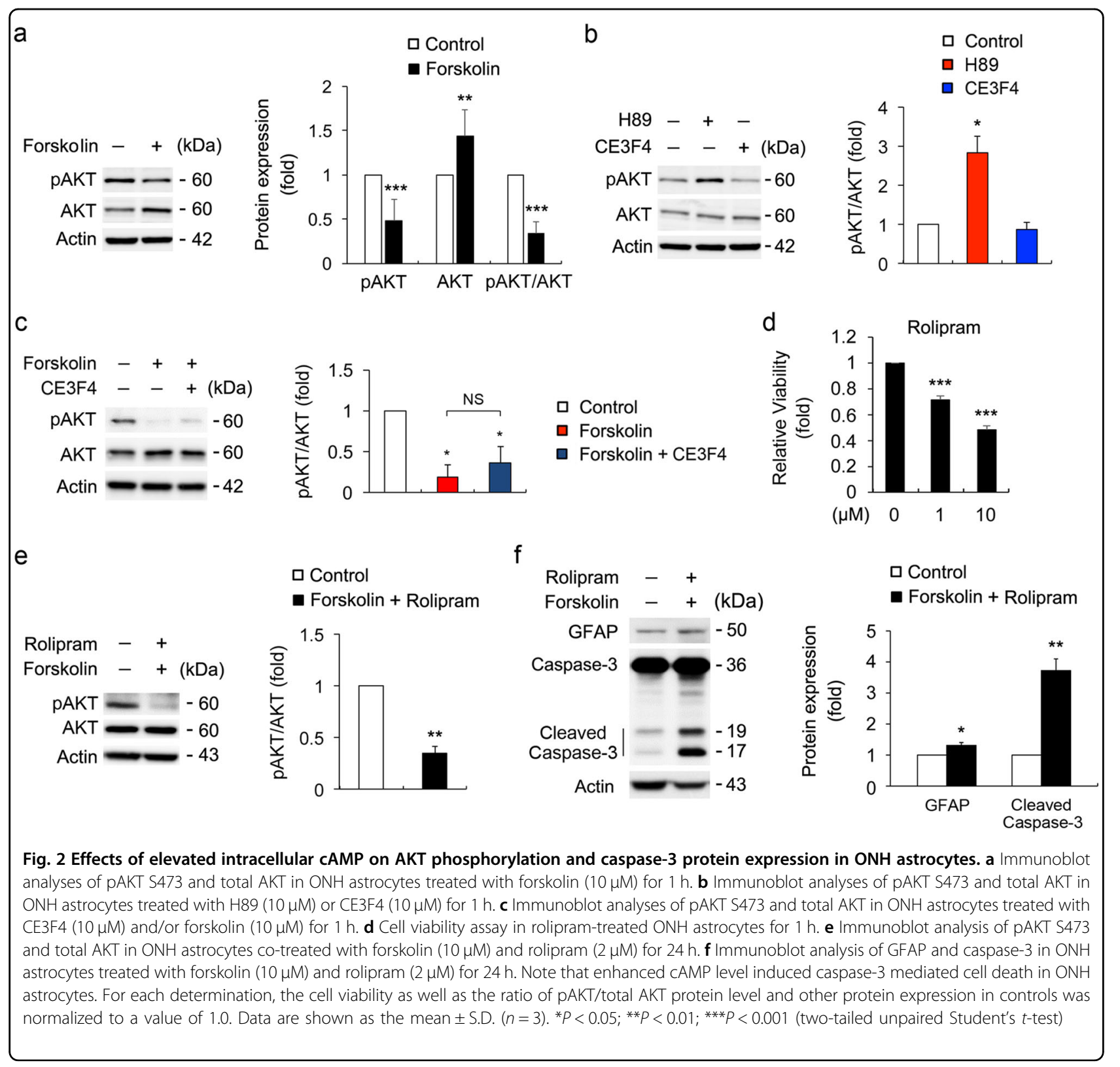

stresses $^{22}$. Quantitative RT-PCR analyses demonstrated that $\mathrm{H}_{2} \mathrm{O}_{2}$ treatment alone induced a significant increase of the expression level of mature TNF $\alpha$ mRNA in ONH astrocytes in a dose-dependent manner, in contrast to a significant decrease in the expression level of precursor transcripts of TNF $\alpha$ mRNA (Fig. 6d). Interestingly, forskolin treatment alone also induced the reciprocal shifts in the expression levels of precursor and mature TNF $\alpha$ mRNA in ONH astrocytes by decreasing precursor TNF $\alpha$ mRNA but by increasing mature TNF $\alpha$ mRNA (Fig. 6e). More importantly, forskolin treatment in $\mathrm{H}_{2} \mathrm{O}_{2}$-treated $\mathrm{ONH}$ astrocytes induced the maximum shift from the precursor to mature TNF $\alpha$ mRNA level (Fig. 6e).
However, surprisingly, oxidative stress significantly decreased the intracellular cAMP level and PKA activity in $\mathrm{ONH}$ astrocytes, accompanying by increased ROS production and reduced cell viability compared with the controls (Figs. 7a-d). Consistently, immunocytochemistry analysis confirmed that cAMP immunoreactivity was decreased in the cytoplasm of oxidative stress-induced $\mathrm{ONH}$ astrocytes (Fig. 7e). Among candidates for the PKA target genes (nur77, areg, nr4a2, il-6, rgs2, and dusp1), which are known as specific target genes of the cytosolic PKA pathway ${ }^{23,24}$, the expression level of nur77 and dusp1 mRNA was upregulated by $\mathrm{H}_{2} \mathrm{O}_{2}$ treatment alone but not by $\mathrm{H}_{2} \mathrm{O}_{2}$ and forskolin together, while areg mRNA 


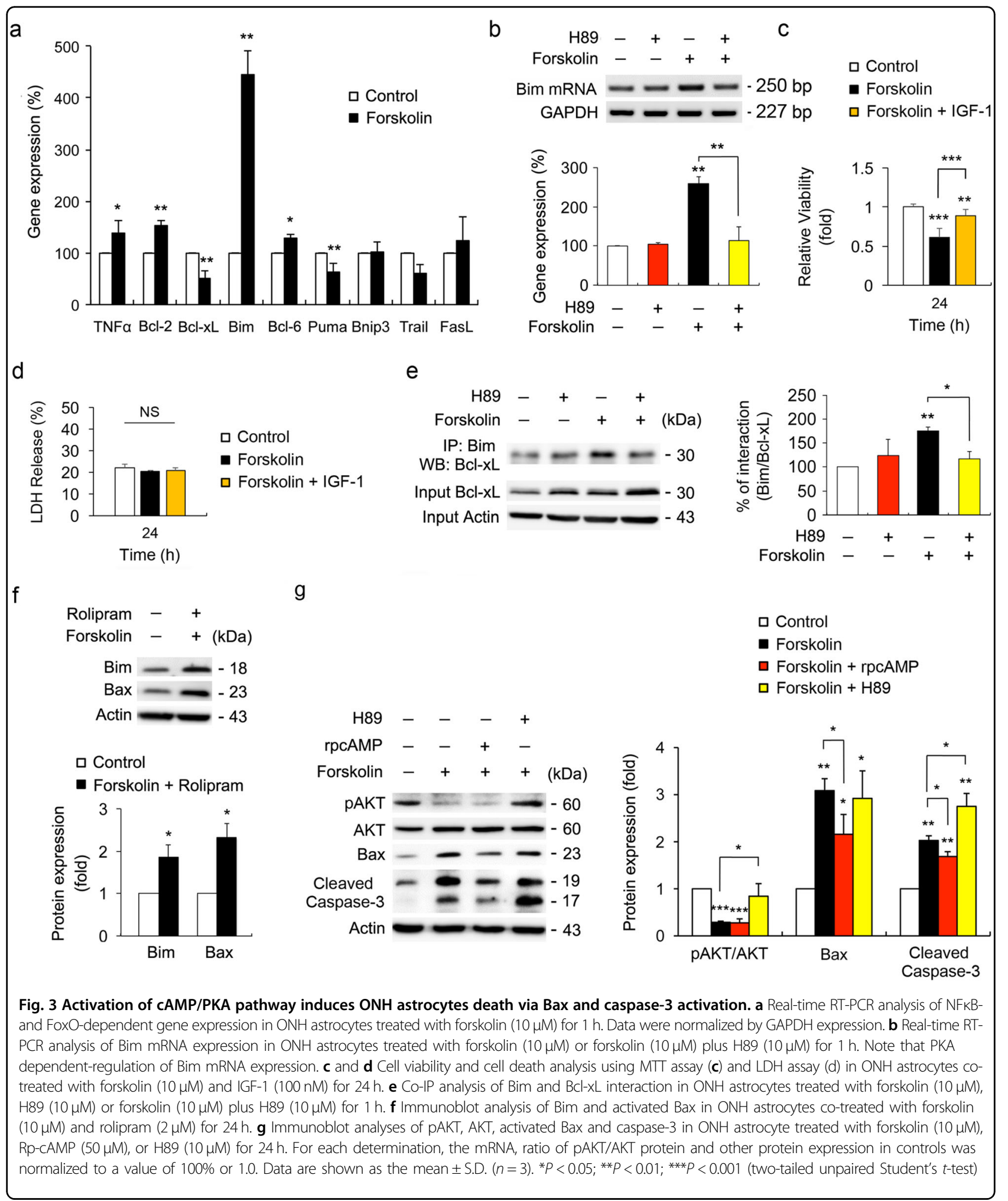

expression was not changed in both $\mathrm{H}_{2} \mathrm{O}_{2}$ - and forskolintreated $\mathrm{ONH}$ astrocytes (Supplementary Fig. S5). Realtime quantitative RT-PCR analysis demonstrated that forskolin treatment alone significantly upregulated the expression levels of rgs 2 , il-6, and $n r 4 a 2$ mRNA in $\mathrm{ONH}$ astrocytes (Fig. 7f). However, the increased level of these genes was significantly decreased in $\mathrm{ONH}$ astrocytes co-treated with $\mathrm{H}_{2} \mathrm{O}_{2}$ and forskolin (Fig. 7f). In addition, 


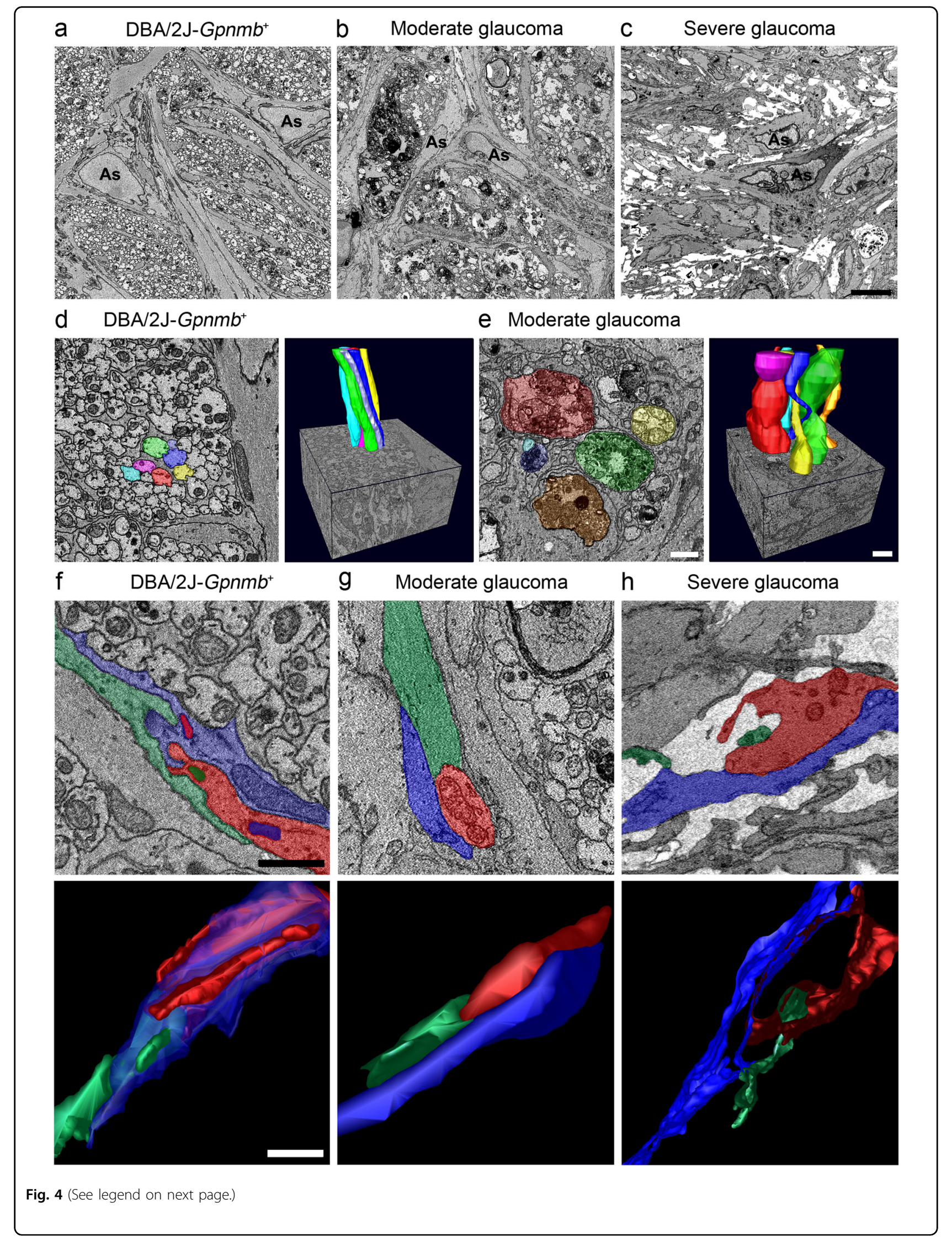




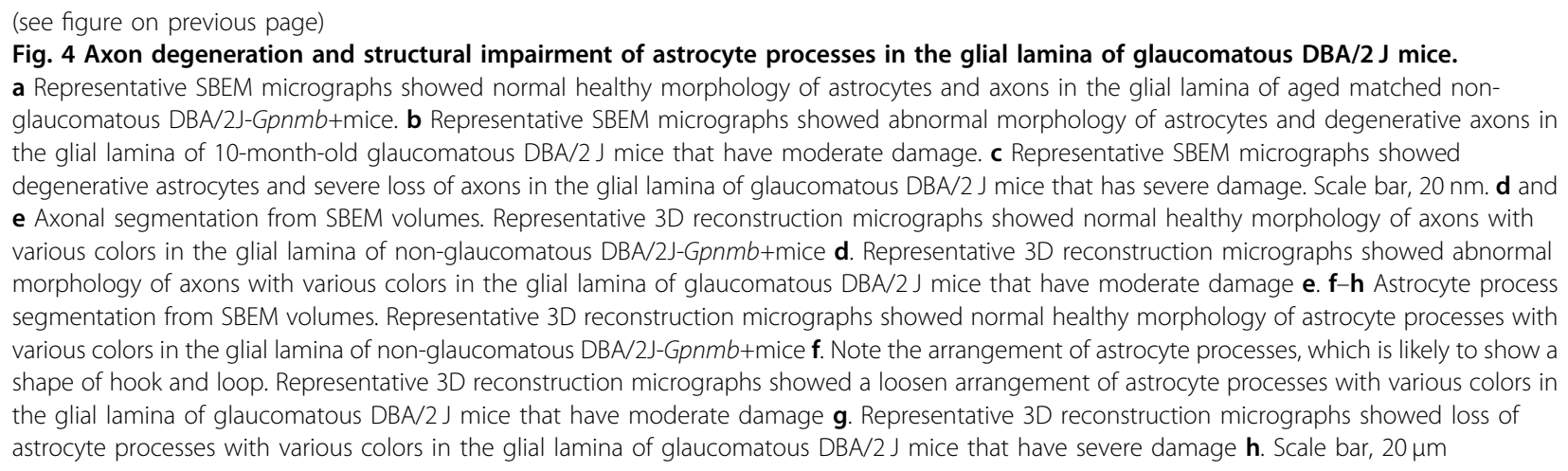

oxidative stress significantly increased the expression level of pAKT S473 (Fig. 7g) and decreased the expression levels of Bim mRNA and protein in ONH astrocytes cotreated with forskolin (Fig. 7h). Our results also showed that there was no statistically significant difference in the expression levels of $A D C Y 10(A C 10)$ mRNA as well as sAC protein in cytosolic and mitochondrial fractions in $\mathrm{ONH}$ astrocytes treated with $\mathrm{H}_{2} \mathrm{O}_{2}$ (Supplementary Fig. S6).

\section{Inhibition of cAMP/PKA pathway protects ONH astrocytes against oxidative stress}

Using Rp-cAMP or transduction of adeno-associated virus serotype 2/5 (AAV2/5)-protein kinase inhibitor (PKI) that block PKA activity, we pretreated Rp-cAMP or overexpressed PKI in ONH astrocytes. We found that PKA inhibition by Rp-cAMP or PKI overexpression significantly promoted cell viability in $\mathrm{ONH}$ astrocytes against oxidative stress (Figs. 8a and b). In addition, either $\mathrm{H}_{2} \mathrm{O}_{2}$ treatment or PKI overexpression alone significantly increased the expression level of pAKT S473 in $\mathrm{ONH}$ astrocytes compared with control cells (Fig. 8c). Surprisingly, the pAKT S473 was highest in PKI-expressing ONH astrocytes treated with $\mathrm{H}_{2} \mathrm{O}_{2}$ (Fig. 8c). However, there were no statistically significant differences in the expression levels of Bim and Bax proteins (Fig. 8c). Thus, elevated intracellular cAMP is critically involved in $\mathrm{ONH}$ astrocyte dysfunction via PKA activation-mediated AKT/ $\mathrm{Bim} /$ Bax pathway, leading to caspase- 3 activation and in turn, exacerbates cell death in oxidative stress (Fig. 8d).

\section{Discussion}

In the present study, we observed that cAMP/PKA pathway inactivated AKT survival pathway in $\mathrm{ONH}$ astrocytes and subsequently this activated FoxO-mediated Bim/Bax death pathway, leading to caspase-3 activationmediated cell death. Notably, elevated cAMP exacerbated the vulnerability to oxidative stress in $\mathrm{ONH}$ astrocytes through the PKA pathway. Another intriguing finding was that inhibition of the PKA pathway also protected $\mathrm{ONH}$ astrocytes against oxidative stress. We therefore propose that the inhibition of cAMP/PKA signaling pathway can be an important therapeutic target for ameliorating astrocyte dysfunction in glaucomatous $\mathrm{ONH}$ degeneration.

cAMP signaling pathway is critically involved in a variety of cellular function including cell survival and death $^{8,9,25,26}$. However, little is known about the related pathophysiological mechanism and its significance on the activated cAMP signaling pathway in dysfunctional glial cells, including $\mathrm{ONH}$ astrocytes, in neurodegeneration. Among the main downstream effectors of cAMP signaling, PKA and Epac have a similar affinity to $\mathrm{CAMP}^{27}$ and contribute to cell growth and death ${ }^{8}$. Recent evidence showed that activation of cAMP/PKA pathway has a critical role in the apoptosis in cancer cell lines ${ }^{28}$ and vascular smooth muscle cells ${ }^{29}$. In contrast, reduced cAMP protects cultured rat cortical astrocytes via the activating AKT pathway ${ }^{30-32}$. Our results demonstrated that activation of cAMP/PKA pathway but not the Epac pathway diminished phosphorylation of AKT S473 in ONH astrocytes. The enhancement of cAMP/PKA activity induced caspase- 3 activation-mediated cell death in $\mathrm{ONH}$ astrocytes. Taken together, these findings raise the possibility that inactivation of AKT by activation of cAMP/ PKA pathway may be an important signal pathway in $\mathrm{ONH}$ astrocyte dysfunction.

AKT pathway controls the expression of apoptosisrelated genes through modulating transcription factors such as $\mathrm{NF}-\mathrm{KB}^{33,34}$ and $\mathrm{FoxO}^{35,36}$. Of note, our results showed that elevated intracellular cAMP induced a substantial increase of Bim expression, which is regulated by FoxO3a transcription factor $^{35,37}$ in $\mathrm{ONH}$ astrocytes. Moreover, this upregulation of Bim expression by elevated cAMP was suppressed by PKA inhibition or AKT activation. This provides evidence that the activated cAMP/PKA pathway is linked to FoxO-mediated Bim activation in $\mathrm{ONH}$ astrocytes. The pro-apoptotic Bcl-2 homology domain 3-only protein Bim induces apoptosis, primarily 


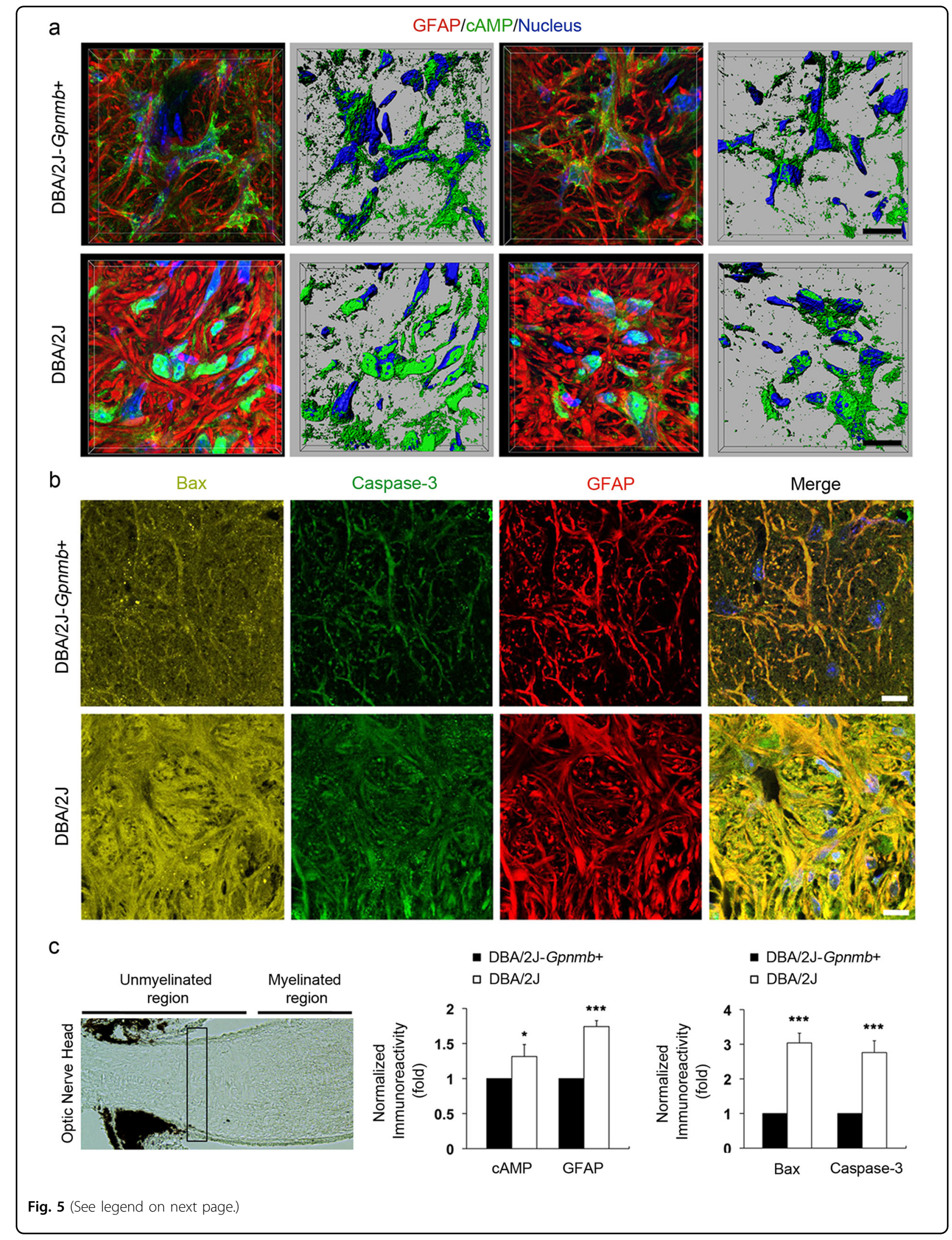




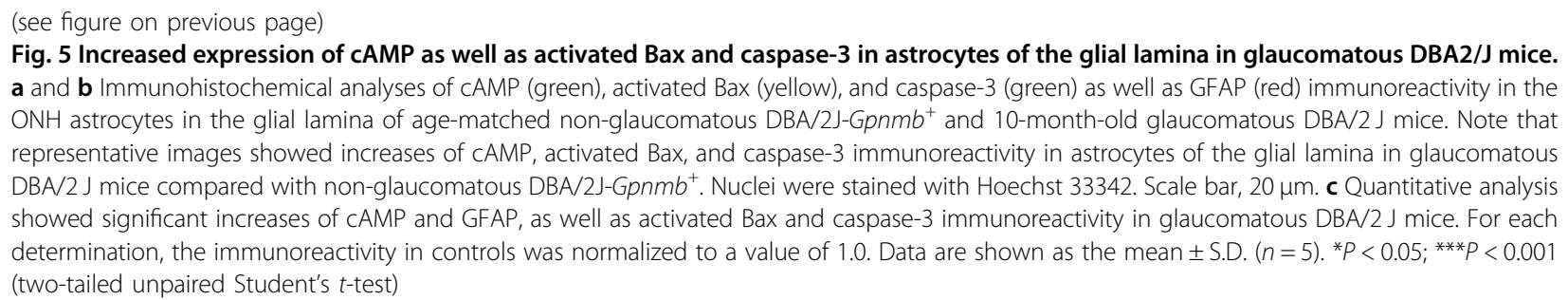

through its increased binding activity toward multiple prosurvival Bcl-2-like proteins ${ }^{38}$, whose dissociations activate $\mathrm{Bax}$ and $\mathrm{Bak}^{39}$. Indeed, we demonstrated that elevated cAMP promoted a direct interaction between Bim and $\mathrm{Bcl}-\mathrm{xL}$ in $\mathrm{ONH}$ astrocytes, and this interaction was blocked by PKA inhibition. As Bax activation leads to caspase-3 activation in the mitochondria-related intrinsic apoptosis signaling pathway ${ }^{40}$, longer stimulation of cAMP increased activated Bax and caspase- 3 in $\mathrm{ONH}$ astrocytes. These findings strongly suggest the possibility that elevated cAMP contributes to cellular dysfunction through AKT inactivation and $\mathrm{Bim} / \mathrm{Bax}$ activation, leading to intrinsic apoptosis signaling pathway, in $\mathrm{ONH}$ astrocytes.

We demonstrated that PKA inhibition by Rp-cAMP significantly inhibited activation of both Bax and caspase3 , whereas Rp-cAMP could not restore AKT phosphorylation in forskolin-treated $\mathrm{ONH}$ astrocytes. Interestingly, however, PKA inhibition by $\mathrm{H} 89$ restored AKT phosphorylation, whereas H89 could not prevent activation of both Bax and caspase-3 activation in forskolin-treated $\mathrm{ONH}$ astrocytes. It is possible that prolonged activation of PKA might not completely be prevented by Rp-cAMP because it is a competitive inhibitor of the cAMP-binding site of PKA regulatory subunits and its effect is likely to be diminished when endogenous levels of cAMP are extremely high ${ }^{41}$. This notion is also supported by the effect of PKI overexpression on pAKT expression under prolonged PKA activation by forskolin and rolipram treatment for 24h. Although pAKT expression was increased in PKIoverexpressing $\mathrm{ONH}$ astrocytes compared with control cells, PKI did not restore the reduction of pAKT expression. However, interestingly, PKI significantly decreased Bax activation (Supplementary Fig. S7), showing a similar pattern of the effect of Rp-cAMP on PKA. As PKI specifically inhibits PKA activity by binding to the free catalytic subunit, which mimics the function of regulatory subunit of $\mathrm{PKA}^{42}$, it is also not sufficient to block the prolonged activation of PKA completely like as Rp-cAMP. However, H89 inhibits catalytic subunits of PKA directly to competing with ATP and it could block completely the prolonged activation of PKA more efficiently than RpcAMP and $\mathrm{PKI}^{43}$. Furthermore, H89 also inhibits nonspecifically other kinases including Rho-associated kinase in a PKA-independent manner ${ }^{41}$. Therefore, the pharmacological drugs that modulate the regulatory subunit of PKA may be more attractive than the drugs that modulate catalytic subunit activity for promoting preventive effect.

As increased cAMP is associated with the promotion of neurotrophic sensitivity in RGC survival ${ }^{44,45}$, recent evidence suggested that RGC survival and axon growth are enhanced by the activation of $\mathrm{sAC}$-generated CAMP $^{9,25}$ but not likely to be tmACs dependent by examining the effects of tmACs inhibitors or the AC1/AC8 double knockout mice ${ }^{25}$. These results suggested that sACgenerated cAMP has a critical role for RGC survival and axon growth, whereas tmACs-generated cAMP has a minor effect on RGC survival and axon growth. Surprisingly, however, our results demonstrated that elevated cAMP by forskolin treatment showed a beneficial effect by promoting cell survival in RGCs. In contrast, pharmacological inhibition of cAMP/PKA activation lowered the cell viability in RGCs. Based on these results demonstrated here, it is possible that elevated cAMP has distinctive roles in the cellular processes of RGC axons and astrocytes in glaucomatous ONH degeneration. Previous studies have reported that glaucomatous damage induced increase of two tmACs, $A C 3$ and $A C 9$, gene expression in the unstimulated $\mathrm{ONH}$ astrocytes from AA donors with POAG as well as in the elevated hydrostatic pressureinduced $\mathrm{ONH}$ astrocytes from normal AA donors ${ }^{11,12}$. Consistently, these results were supported by the observation of increased expression levels of cAMP, Bax and caspase- 3 protein in the astrocytes of the glial lamina in glaucomatous DBA/2 J mice, accompanied by structural loss of astrocytic processes and degenerative axons. Together, these results suggest another possibility that elevated cAMP-dependent pathway is an important player in not only $\mathrm{ONH}$ astrocytes but also RGC axons against glaucomatous insults such as elevated IOP and oxidative stress. Collectively, our results identify for the first time a pathological link between cAMP/PKA pathway activation and $\mathrm{AKT} / \mathrm{Bim} / \mathrm{Bax}$-mediated intrinsic cell death pathway in $\mathrm{ONH}$ astrocyte dysfunction.

Oxidative stress is considered as a key risk factor in many neurodegenerative diseases including glaucoma $^{2,4,13,15}$. Our results show that elevated cAMP 


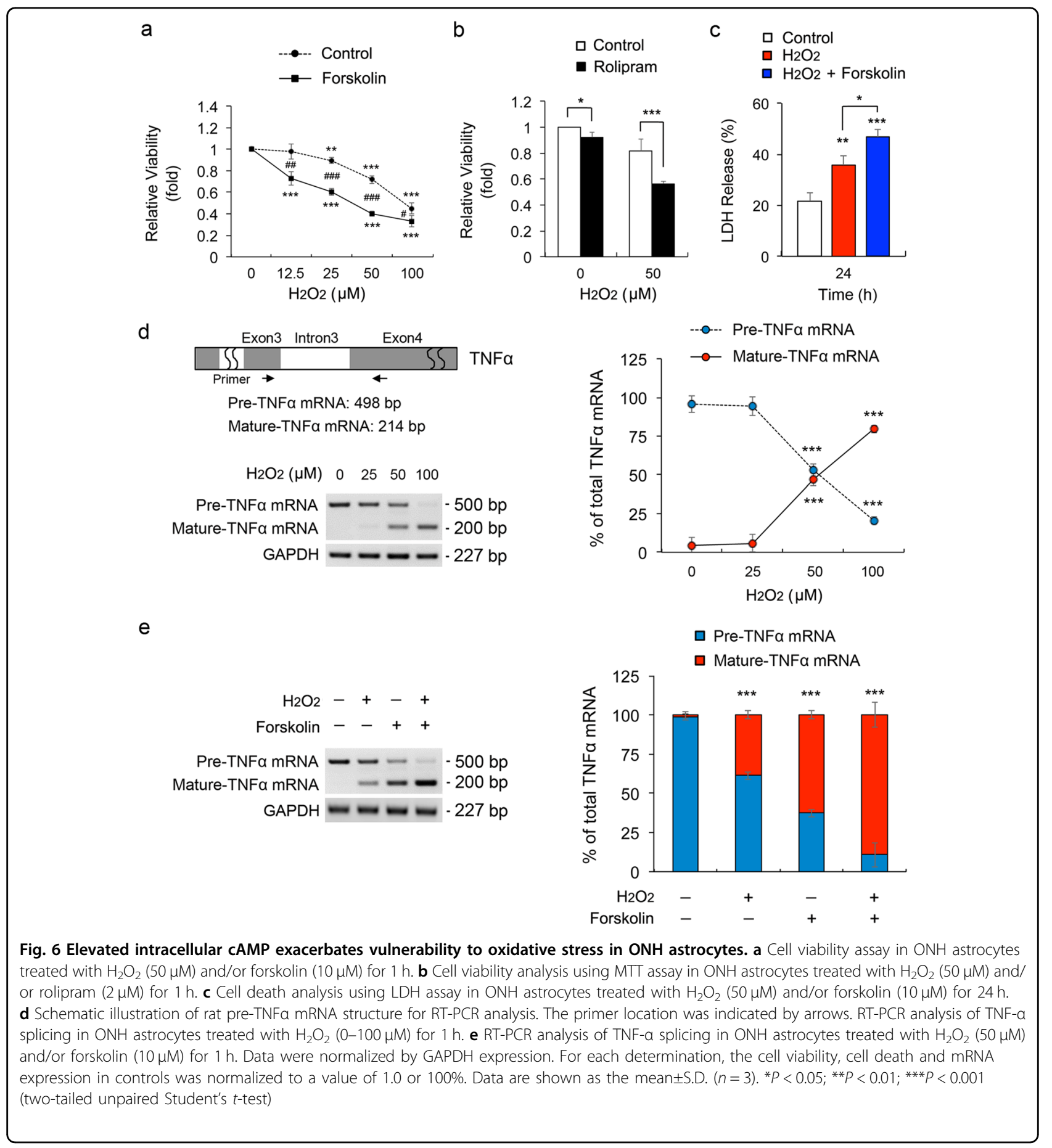

exacerbated the vulnerability of $\mathrm{ONH}$ astrocytes against oxidative stress. As a well-known molecular biomarker in POAG $^{46,47}$, an increase of TNF $\alpha$ expression has been reported in glial cells of the $\mathrm{ONH}$ from patients with glaucoma $^{48-50}$, and glial cell-derived TNF $\alpha$ signaling pathway is associated with oxidative stress and RGC axon degeneration in glaucomatous $\mathrm{ONH}^{48,50,51}$. In the present study, our results demonstrated that elevated intracellular
cAMP may not only activate TNF $\alpha$ by inducing the splicing of TNF $\alpha$ mRNA in ONH astrocytes but also accelerated TNF $\alpha$ activation by enhancing the splicing of TNF $\alpha$ mRNA in ONH astrocyte in synergy with oxidative stress. On the other hand, intriguingly, our results also demonstrated that oxidative stress alone induced the reduction of cAMP level and PKA activity in $\mathrm{ONH}$ astrocytes. Therefore, our findings support the notion that 


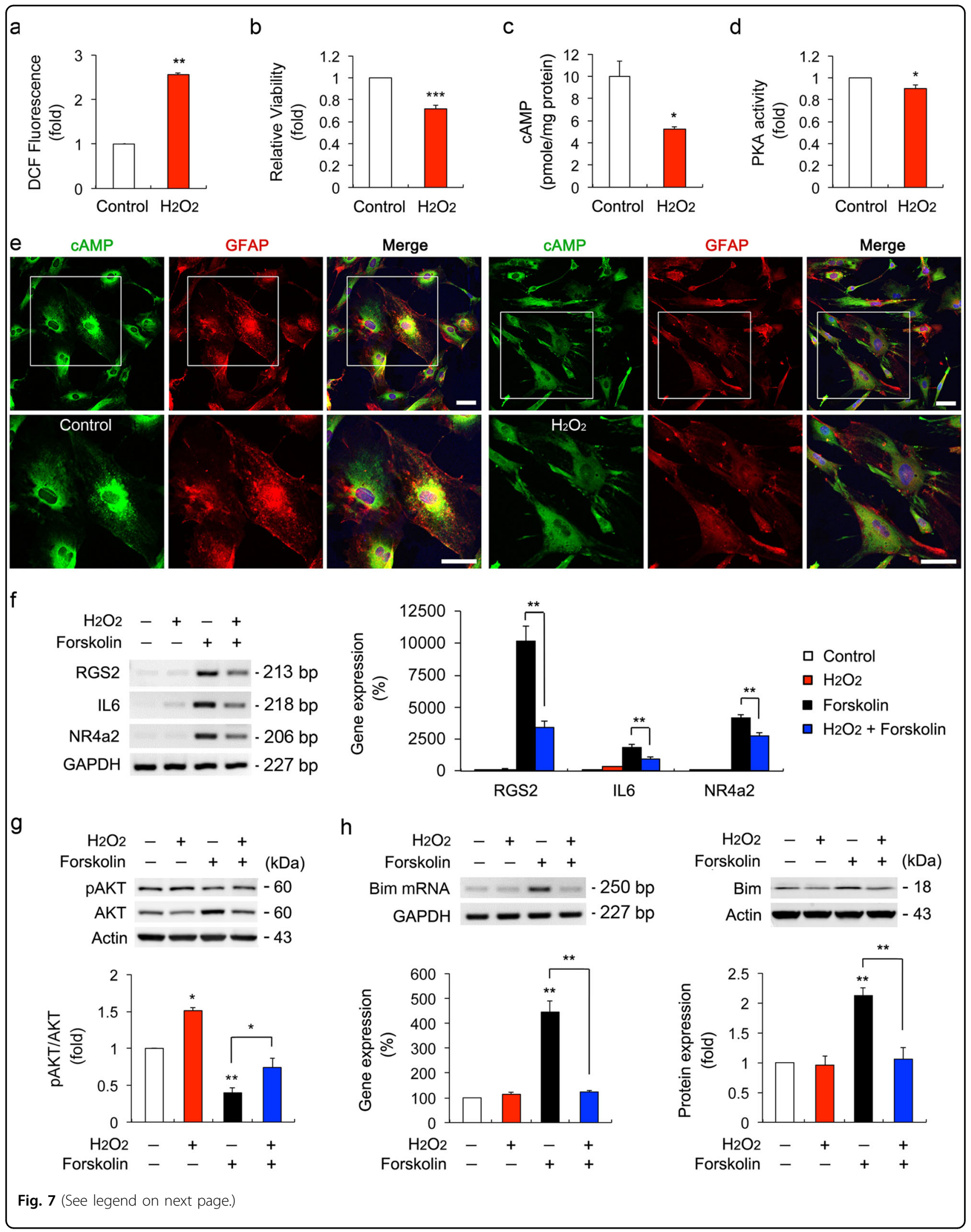


(see figure on previous page)

Fig. 7 Effects of oxidative stress on CAMP/PKA signaling pathway in ONH astrocytes. a ROS production in ONH astrocytes treated with $\mathrm{H}_{2} \mathrm{O}_{2}(50 \mu \mathrm{M})$ for $1 \mathrm{~h}$. b Cell viability analysis using MTT assay in ONH astrocytes treated with $\mathrm{H}_{2} \mathrm{O}_{2}(50 \mu \mathrm{M})$ for $1 \mathrm{~h}$. c Intracellular cAMP measurement in $\mathrm{ONH}$ astrocytes treated with $\mathrm{H}_{2} \mathrm{O}_{2}(50 \mu \mathrm{M})$ for $1 \mathrm{~h}$. d PKA activity measurement in ONH astrocytes treated with $\mathrm{H}_{2} \mathrm{O}_{2}(50 \mu \mathrm{M})$ for $1 \mathrm{~h}$. For each determination, the ROS, cell viability, PKA activity and mRNA expression in controls was normalized to a value of 1.0 and the cAMP level was normalized to the protein contents. e Immunocytochemical analyses of cAMP and GFAP in ONH astrocytes treated with $\mathrm{H}_{2} \mathrm{O}_{2}(50 \mu \mathrm{M})$ for 1 h. Representative images showed a decrease of CAMP immunoreactivity in $\mathrm{H}_{2} \mathrm{O}_{2}$-treated ONH astrocytes. Note that decreased level of cAMP in the cytoplasm of ONH astrocytes treated with $\mathrm{H}_{2} \mathrm{O}_{2}(50 \mu \mathrm{M})$. Scale bars, $20 \mu \mathrm{m}$. $\mathbf{f}$ Real-time RT-PCR analysis of PKA target genes in ONH astrocytes treated with $\mathrm{H}_{2} \mathrm{O}_{2}(50 \mu \mathrm{M})$ and/or forskolin $(10 \mu \mathrm{M})$ for $1 \mathrm{~h}$. Data were normalized by GAPDH expression. $\mathbf{g}$ Immunoblot analyses of pAKT and total AKT in ONH astrocytes treated with $\mathrm{H}_{2} \mathrm{O}_{2}(50 \mu \mathrm{M})$ and/or forskolin $(10 \mu \mathrm{M})$ for $1 \mathrm{~h}$. For each determination, the ratio of pAKT/total AKT protein level in control cells with no treatment was normalized to a value of 1.0. $\mathbf{h}$ Real-time RT-PCR and immunoblot analyses of Bim in ONH astrocytes treated with $\mathrm{H}_{2} \mathrm{O}_{2}(50 \mu \mathrm{M})$ and/or forskolin (10 $\left.\mu \mathrm{M}\right)$ for $1 \mathrm{~h}$. Data were normalized by GAPDH and Actin expression, respectively. For each determination, the mRNA and protein expression in controls was normalized to a value of $100 \%$ or 1.0 . Data are shown as the mean \pm S.D. $(n=3) .{ }^{*} P<0.05 ;{ }^{* *} P<0.01 ;{ }^{* * *} P<0.001$ (two-tailed unpaired Student's t-test)

elevated cAMP-mediated enhancement of TNF $\alpha$ activation could be critical for oxidative stress-mediated $\mathrm{ONH}$ astrocyte dysfunction and RGC axon degeneration in POAG.

On the other hand, intriguingly, our results also demonstrated that oxidative stress alone decreased cAMP level and PKA activity and subsequently activated AKT pathway in $\mathrm{ONH}$ astrocytes. How is this possible? In the central nervous system, neurons are most vulnerable cells to oxidative stress due to their low ROS- detoxifying capacity, therefore its survival is highly dependent on the capacity of neighboring astrocytes during oxidative stressinduced neurodegeneration ${ }^{52,53}$. Emerging evidence suggests that AKT activity is critical for astrocyte resilience to oxidative stress and enhances the protective effect of astrocytes against oxidative stress ${ }^{54}$. Indeed, we observed that oxidative stress activated AKT pathway in $\mathrm{ONH}$ astrocytes, accompanied by restoration of Bim expression. Because a rapid and transient increase in relatively lower concentration of $\mathrm{H}_{2} \mathrm{O}_{2}(25 \mu \mathrm{M})$ promotes the pro-survival activity in photoreceptor cells against serum deprivation by increasing AKT phosphorylation ${ }^{55}$, our results raise a possibility that a transient induction of oxidative stress (relatively low concentration of $\mathrm{H}_{2} \mathrm{O}_{2}$ ) in $\mathrm{ONH}$ astrocytes may trigger the reduction of cAMP level and PKA activity by a rapid and transient activation of AKT survival pathway, which in turn, it may inhibit Bim activation. In addition, our results also demonstrated that enhanced AKT phosphorylation by IGF-1 rescued ONH astrocytes against elevated cAMP. These results suggest that increasing AKT phosphorylation may be an important endogenous compensatory defense mechanism against oxidative stress, which is mediated by inactivation of cAMP/PKA signaling pathway, in $\mathrm{ONH}$ astrocytes. Therefore, the dysregulation of the defense system by elevated cAMP level may accelerate $\mathrm{ONH}$ astrocyte dysfunction in oxidative stress.

Recent evidence has demonstrated that inhibition of PKA pathway by $\mathrm{H} 89$ protected hippocampus against A $\beta$ induced oxidative stress via increases of glutathione levels and superoxide dismutase activity ${ }^{56}$. Furthermore, antiapoptotic markers, such as Bcl-xL, pBad, and heat shock protein 70 , are increased in the brain of aged AC5 knockout mouse that shows resistance against oxidative stress $^{57}$. Our results indicated that PKA inhibition by Rp-cAMP treatment promoted cell viability in $\mathrm{ONH}$ astrocytes against oxidative stress. Furthermore, PKI overexpression protected $\mathrm{ONH}$ astrocytes against oxidative stress by enhancing AKT phosphorylation. Taken together, these results further support the notion that activation of cAMP/PKA pathway may exacerbate vulnerability to oxidative stress in $\mathrm{ONH}$ astrocytes and consequently may promote susceptibility to the factors contributing to progressive axon degeneration in POAG.

In summary, we provide evidence that the cAMP/PKA signaling pathway is important to the vulnerability of oxidative stress-induced $\mathrm{ONH}$ astrocytes and that inhibition of intracellular cAMP/PKA signaling activation may protect $\mathrm{ONH}$ astrocytes by increasing AKT phosphorylation against oxidative stress. These results suggest that modulating cAMP/PKA signaling pathway has therapeutic potential for glaucoma pathogenesis.

\section{Methods}

\section{Animals}

Pregnant Sprague-Dawley rats $(250-300 \mathrm{~g}$ in weight; Harlan Laboratories) and adult female DBA/2 J and DBA/ 2J-Gpnmb ${ }^{+}$mice (The Jackson Laboratory) were housed in covered cages, fed with a standard rodent diet $a d$ libitum, and kept on a $12 \mathrm{~h}$ light $/ 12 \mathrm{~h}$ dark cycle. All procedures concerning animals were in accordance with the Association for Research in Vision and Ophthalmology Statement for the Use of Animals in Ophthalmic Vision Research and under protocols approved by Institutional Animal Care and Use Committee at the University of California, San Diego.

\section{Reagents and other materials}

Chemicals and other materials were obtained from the following sources: $\mathrm{H}_{2} \mathrm{O}_{2}$, forskolin, Rp-cAMP, and H89 


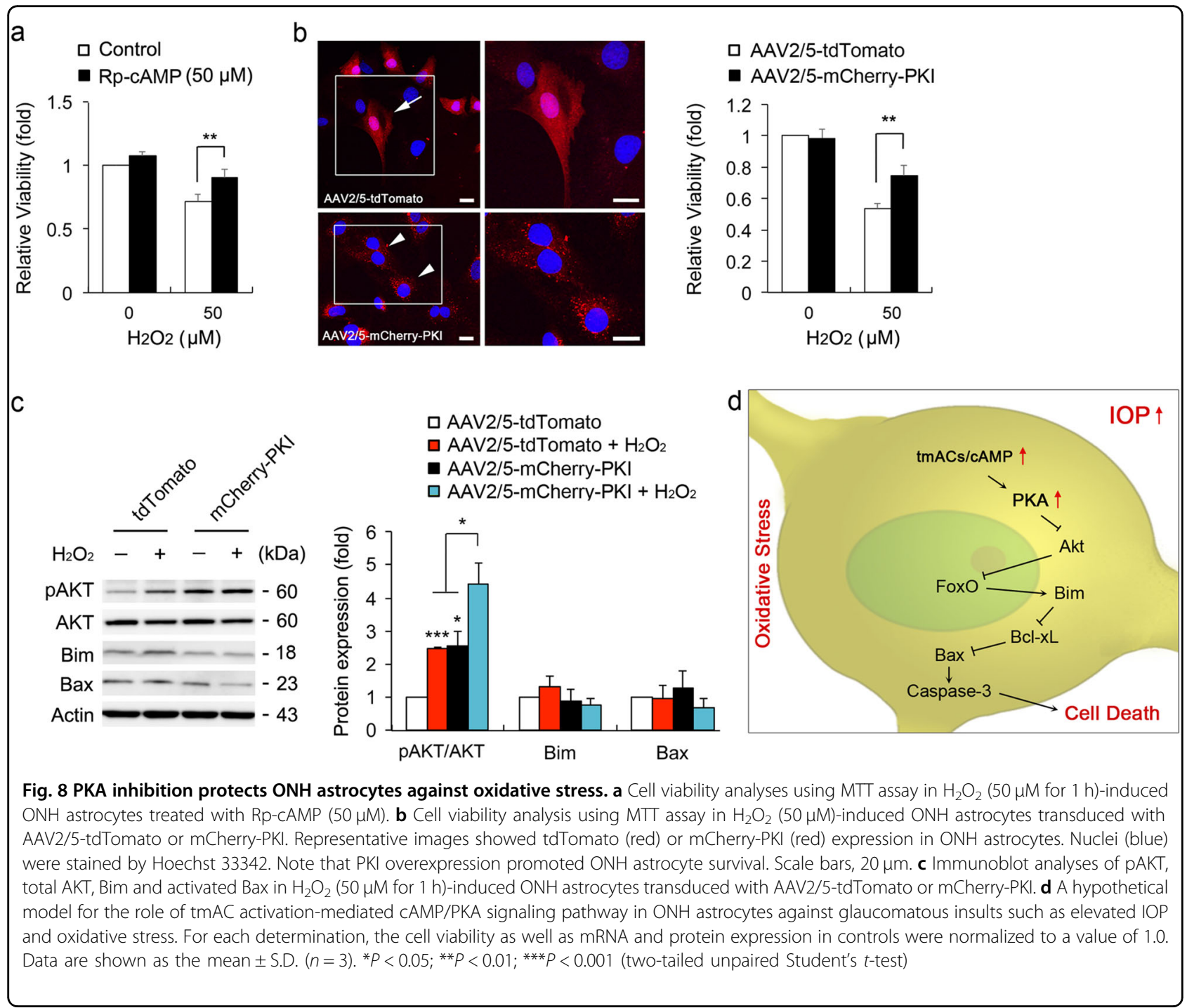

from Sigma; Rolipram from Cayman Chemical; Recombinant Rat IGF-1 from Prospec; DMEM/F12 (1:1), MEM +GlutaMax, $100 \times$ penicillin-streptomycin (Pen/Strep) and fetal bovine serum from Gibco; Superscript II reverse transcriptase from Invitrogen; RNase inhibitor, dNTP mixture and oligo (dT) from Promega; PCR master mix, protein size marker, SuperSignal West Pico and Femto chemiluminescent substrates from Thermo scientific; Amersham Hybond-P (PVDF membrane) and ECL Prime Western Blotting Detection Reagent from GE Healthcare; Lab-Tek Chambered cover glass from Nunc; CE3F4 from TOCRIS.

\section{Tissue preparation}

Mice were anesthetized with intraperitoneal injection of a mixture of ketamine $(100 \mathrm{mg} / \mathrm{kg}$, Ketaset; Fort Dodge Animal Health) and xylazine $(9 \mathrm{mg} / \mathrm{kg}$, TranquiVed; VEDCO Inc.) before cervical dislocation. For immunohistochemistry, the retinas and $\mathrm{ONHs}$ were dissected from the choroids and fixed for $2 \mathrm{~h}$ at $4{ }^{\circ} \mathrm{C}$ with $4 \%$ paraformaldehyde (Sigma) in phosphate-buffered saline (PBS, pH 7.4). After several washes in PBS, the retinas were dehydrated through graded ethanols and embedded in polyester wax. For immunoblot analyses, extracted retinas were immediately used.

\section{Primary ONH astrocyte culture}

Primary rat $\mathrm{ONH}$ astrocytes were prepared as described previously with minor modifications ${ }^{16}$. In brief, after euthanizing with $\mathrm{CO}_{2}$ gas, 10-20 pieces of $\mathrm{ONH}$ tissue were dissected from postnatal day 5 Sprague-Dawley rats and transferred to a $35-\mathrm{mm}$ petri dish with $2 \mathrm{ml} 0.2 \%$ bovine serum albumin (BSA)/Dulbecco's phosphatebuffered saline (DPBS). Under a dissecting microscope, remnant tissues were removed, and the $\mathrm{ONH}$ tissue was identified and dissected using a micro-scissors and sharp blade. The $\mathrm{ONH}$ tissues were minced and transferred to a 
60-mm petri dish and conditioned with growth medium: MEM+GlutaMax (Gibco) supplemented with $10 \%$ fetal bovine serum, $100 \mathrm{U} / \mathrm{ml}$ penicillin and $100 \mu \mathrm{g} / \mathrm{ml}$ streptomycin (Gibco), and incubated at $5 \% \mathrm{CO}_{2}$ at $37^{\circ} \mathrm{C}$. After incubation for 1-2 weeks, the $\mathrm{ONH}$ explants were removed by $70 \mu \mathrm{m}$ cell strainers (BD Biosciences). The cells that were grown from the $\mathrm{ONH}$ explants were plated in a $100 \mathrm{~mm}$ culture dish and cultured until $80 \%$ confluence. For further purification of the $\mathrm{ONH}$ astrocytes, the culture dish was shaken for $16 \mathrm{~h}$ at room temperature, followed by growth medium changing with serum-free medium and the cells were incubated for $24 \mathrm{~h}$ at $5 \% \mathrm{CO}_{2}$ at $37^{\circ} \mathrm{C}$. After removing non-adherent cells, the adherent $\mathrm{ONH}$ astrocytes were collected, centrifuged and plated on poly-L-lysine (PLL)-coated culture dishes.

\section{Primary RGC culture system}

RGCs from postnatal 3-5 days of Sprague-Dawley rats were purified by immunopanning as described previously $^{58,59}$. In brief, $\sim 15,000$ purified cells were seeded on 24-well plates coated first with poly-D-lysine $(10 \mu \mathrm{g} / \mathrm{ml}$; Sigma) and then with laminin $(10 \mu \mathrm{g} / \mathrm{ml}$; Sigma $)$ in neurobasal medium. RGCs were cultured in serum-free defined growth medium containing BDNF $(50 \mu \mathrm{g} / \mathrm{ml}$; PeproTech), CNTF (10 $\mu \mathrm{g} / \mathrm{ml}$; Sigma), insulin $(5 \mu \mathrm{g} / \mathrm{ml}$; Sigma), and forskolin (10 $\mu$ M; Sigma).

\section{Cell viability assay}

Cell viability was measured using MTT according to the manufacturer's recommendations (Cell Proliferation Kit1; Roche Diagnostics). In brief, $\mathrm{ONH}$ astrocytes were plated on a 96-well plate $\left(0.5 \sim 1 \times 10^{4}\right.$ per well). After $48 \mathrm{~h}$, the cells were treated with various reagents for $1 \mathrm{~h}$ and $10 \mu \mathrm{l}$ MTT stock solution was added to each well including the negative control. The cells were incubated for $4 \mathrm{~h}$ in a humidified atmosphere of a $5 \% \mathrm{CO}_{2}$ incubator at $37^{\circ} \mathrm{C}$ and $100 \mu \mathrm{l}$ of solubilization solution were added to dissolve the formazan crystals that remained in the wells. After incubation for overnight at $5 \% \mathrm{CO}_{2}$ at $37^{\circ} \mathrm{C}$, the absorbance at 560 and $690 \mathrm{~nm}$ were measured with a microplate reader (SpectraMAX; Molecular Devices). Each set of data was collected from multiple replicate wells of each experimental group $(n=3)$.

\section{Cell death assay}

Dead and membrane-ruptured cells were quantitatively measured by LDH release assay. LDH activity in cultured medium was assessed with Cytotoxicity Detection Kit (LDH; Sigma) according to the manufacturer's instructions. In brief, After $\mathrm{ONH}$ astrocytes were treated with forskolin $(10 \mu \mathrm{M})$ and/or $\mathrm{H}_{2} \mathrm{O}_{2}(50 \mu \mathrm{M})$ for $24 \mathrm{~h}, 100 \mu \mathrm{l}$ of cell culture medium of each group were mixed with the $100 \mu \mathrm{l}$ reaction mixture in a microplate followed by $30 \mathrm{~min}$ incubation at room temperature in dark environment. The absorbance of the samples was measured at $490 \mathrm{~nm}$ using a microplate leader (SpectraMAX, Molecular Devices). Two percent triton X-100-treated samples were used as positive controls. Each set of data was collected from multiple replicate dishes of each experimental group $(n=3)$.

\section{RT-PCR and quantitative real-time RT-PCR}

In brief, total RNA was isolated from the cells using the RNeasy mini kit (Qiagen), according to the manufacturer's protocol. cDNAs were synthesized from total RNAs with Superscript II reverse transcriptase and oligo (dT) primers according to the manufacturer's protocols. RT-PCR was performed with cDNAs synthesized from $0.1 \mu \mathrm{g}$ of the total RNA of each cell as a template and specific primers (Supplementary Table 1). RT-PCR products were electrophoresed on a $2 \%$ agarose gel and visualized by ethidium bromide staining. For the quantification of the relative mRNA expressions of each group, real-time PCR was carried out using MX3000P real-time PCR system (Stratagene) as follows. cDNAs were amplified using iQ SYBR Green super-mix (Bio-Rad) and the specific primers for 40 cycles (initial incubation at $50^{\circ} \mathrm{C}$ for $2 \mathrm{~min}$ and then at $95^{\circ} \mathrm{C}$ for $10 \mathrm{~min}$, and 40 cycles $\left(95^{\circ}\right.$ $\mathrm{C}$ for $15 \mathrm{sec}, 55^{\circ} \mathrm{C}$ for $1 \mathrm{~min}$, and $72^{\circ} \mathrm{C}$ for $\left.1 \mathrm{~min}\right)$ ). Output data were obtained as $C t$ values and the differential mRNA expression of each gene among samples was calculated using the comparative $C t$ method. GAPDH mRNA, an internal control, was amplified along with the target genes, and the $C t$ value of GAPDH was used to normalize the expression of target genes.

\section{Immunohistochemical and immunocytochemical analyses}

For immunohistochemistry of tissue section, five sections (7 $\mu \mathrm{m}$ thickness) per wax block of ONHs from 10month-old glaucomatous DBA/2 J and age-matched nonglaucomatous DBA/2J-Gpnmb $b^{+}$mice $(n=3$ mice/group) were prepared. Following incubation in 1\% BSA/PBS for $1 \mathrm{~h}$ at room temperature to prevent nonspecific background, the sections were incubated with the primary antibodies for $16 \mathrm{~h}$ at $4{ }^{\circ} \mathrm{C}$. The primary antibodies were mouse monoclonal anti-cAMP antibody (1:500; abcam), guinea pig polyclonal anti-GFAP antibody (1:500; Advanced Immuno Chemical, Inc.), and rabbit polyclonal caspase-3 (1:100; Cell signaling). After several washing, the sections were incubated with the secondary antibodies Alexa Fluor 488 dye-conjugated goat anti-mouse IgG (1:100; Invitrogen) and Cy5-conjugated anti-guinea pig IgG antibody (1:100; Jackson ImmunoResearch Laboratories) for $2 \mathrm{~h}$ at $4{ }^{\circ} \mathrm{C}$ and were subsequently washed with PBS. The sections were counterstained with Hoechst $33342(1 \mu \mathrm{g} / \mathrm{ml}$; Invitrogen $)$ in PBS. For immunocytochemistry of cultured $\mathrm{ONH}$ astrocytes, the cells were fixed with $4 \%$ paraformaldehyde/PBS for overnight at 
$4{ }^{\circ} \mathrm{C}$. After blocking nonspecific background with $1 \%$ BSA/PBS for $1 \mathrm{~h}$ at room temperature, primary antibodies were incubated for $16 \mathrm{~h}$ at $4{ }^{\circ} \mathrm{C}$. The primary antibodies were mouse monoclonal anti-cAMP antibody (1:500; Sigma), mouse monoclonal anti-Bax antibody (6A7; 1:100; Santa Cruz), and rabbit polyclonal caspase-3 (1:100; Cell signaling). After several wash steps, the cells were incubated with the secondary antibody, Alexa Fluor 488 dye-conjugated goat anti-mouse IgG antibody (1:100; Invitrogen) or Alexa Fluor 488 dye-conjugated goat antirabbit IgG antibody (1:100; Invitrogen) for $4 \mathrm{~h}$ at $4{ }^{\circ} \mathrm{C}$ and subsequently washed with PBS. The cells were counterstained with Hoechst $33342(1 \mu \mathrm{g} / \mathrm{ml}$, Life Technologies) in PBS. Images were acquired with confocal microscopy (Olympus FluoView1000; Olympus). ImageJ (http://rsb. info.nih.gov/ij/) was used to measure the fluorescence intensity in pixels per area in each cAMP, GFAP, Bax, and caspase-3 images from glaucomatous DBA/2 J and agematched control DBA/2J-Gpnmb ${ }^{+}$mice. In each antibody image acquisition, all imaging parameters remain the same. Mean pixel intensity was measured in this 11,124 square pixel area.

\section{CAMP measurement}

Cells $\left(2 \sim 5 \times 10^{5}\right)$ were washed in ice-cold PBS, harvested by centrifugation at $1800 \mathrm{~g}$ for $5 \mathrm{~min}$ and extracted with $0.1 \mathrm{M} \mathrm{HCl}$. The extracts were centrifuged at $15,000 \mathrm{~g}$ for $3 \mathrm{~min}$ and the supernatants were collected. Intracellular cAMP levels in the final supernatants were measured by using a direct cAMP ELISA kit (Enzo Life Sciences) according to the manufacturer's instructions for acetylation format. Protein concentration of the same samples was determined by Bradford protein quantification assay. Data were normalized to the protein amounts.

\section{ROS measurement}

The intracellular ROS was measured by using cell permeant reagent 2',7'-dichlorofluorescin diacetate (DCFHDA, Sigma). In brief, ONH astrocytes were plated on a PLLcoated six-well plate $\left(2 \times 10^{4}\right.$ cell per well $)$ and after $24 \mathrm{~h}$, cells were exposed to $50 \mu \mathrm{M}$ of $\mathrm{H}_{2} \mathrm{O}_{2}$ for $3 \mathrm{~h}$ in serum-free condition. The cells were added with $200 \mathrm{nM}$ of DCFHDA, incubated at $37^{\circ} \mathrm{C}$ for $20 \mathrm{~min}$ and then detached with $0.25 \%$ typsin/EDTA. After washing with DPBS, the fluorescence intensity of the sample was measured immediately using C6 Accuri flow cytometer (BD Biosciences). Each set of data was collected from multiple replicate dishes of each experimental group $(n=3)$.

\section{PKA activity assay}

PKA activity in the samples from $\mathrm{ONH}$ atrocytes was measured by using a non-radioactive PKA activity assay kit (Enzo Life Sciences). In brief, ONH astrocytes $\left(2 \times 10^{5}\right)$ were seeded on PLL-coated $200 \mathrm{~mm}$ dishes in normal growth medium for $48 \mathrm{~h}$. The cells were treated with indicated reagents in serum-free condition for $1 \mathrm{~h}$. Total cellular proteins were extracted by using lysis buffer according to the manufacturer's instruction. PKA substrate-coated microtiter plate was soaked with kinase assay dilution buffer for $10 \mathrm{~min}$ at room temperature. One hundred nano-gram in $30 \mu \mathrm{l}$ of cell lysates or PKA standard (10 ng) were then added, followed by the addition of ATP to initiate the kinase reaction. After incubation at $30^{\circ} \mathrm{C}$ for $90 \mathrm{~min}$, the reaction was terminated by removing the mixture from the plate, and phosphor specific substrate antibody was added to each well and incubated at room temperature for $60 \mathrm{~min}$. The solution was removed completely and the wells were repeatedly washed. The peroxidase-conjugated secondary anti-rabbit IgG was then added to each well and incubated for another $30 \mathrm{~min}$ at room temperature. After several washing, the color was developed with tetramethylbenzidine substrate for 30-60 min and the reaction was stopped by adding acid-stop solution. The absorbance was measured at $450 \mathrm{~nm}$ with a microplate reader (SpectraMAX; Molecular Devices). The absorbance was divided by the concentration of total protein $(\mu \mathrm{g})$ in each sample, and the data are represented as relative PKA activity.

\section{Co-IP and immunoblot analyses}

For co-IP, the cell lysates were prepared in co-IP lysis buffer $(20 \mathrm{mM}$ Tris-Cl, pH 7.4, $135 \mathrm{mM} \mathrm{NaCl}, 1.5 \mathrm{mM}$ $\mathrm{MgCl}_{2}, 1 \mathrm{mM}$ EGTA, 10\% glycerol, 1\% Triton X-100) containing complete protease inhibitors (Roche Biochemicals). IP was performed using Protein A/G PLUSAgarose Immunoprecipitation Reagent (Santa Cruz Biotechnology) and a rabbit polyclonal antibody to Bim (Santa Cruz Biotechnology) according to the manufacturer's instructions. For immunoblot analyses, cells were harvested and lysed for $30 \mathrm{~min}$ on ice with a modified RIPA lysis buffer (150 mM NaCl, $1 \mathrm{mM}$ EDTA, 1\% NP-40, 0.1\% SDS, $1 \mathrm{mM}$ DTT, $0.5 \%$ sodium deoxycholate, and $50 \mathrm{mM}$ Tris- $\mathrm{Cl}, \mathrm{pH} 7.6$ ), containing the complete protease inhibitors. The lysates were centrifuged at $15,000 \mathrm{~g}$ for $15 \mathrm{~min}$ and the protein amounts in the supernatants were measured by Bradford methods. Proteins $(5-10 \mu \mathrm{g})$ were separated by SDS/PAGE and electrotransferred to polyvinylidenedifluoride membrane. The membrane was blocked with $5 \%$ non-fat dry milk and PBS-T (0.1\% Tween-20) for $1 \mathrm{~h}$, incubated with primary antibodies for overnight at $4{ }^{\circ} \mathrm{C}$. Primary antibodies are rabbit polyclonal anti-AKT antibody (1:5000; Cell Signaling), mouse monoclonal anti-pAKT antibody (S473, 1:2000; Cell Signaling), rabbit polyclonal anti-pFoxO1/3 (T24,T32, 1:1,000; cell signaling), rabbit polyclonal anticaspase-3 antibody (1:3000; Cell Signaling), mouse monoclonal anti-Bax antibody (6A7; 1:1000; Santa Cruz Biotechnology), rabbit polyclonal anti-Bcl-xL antibody 
(1:2000; Cell Signaling), mouse monoclonal anti-Bcl-xL antibody (1:1000; Santa Cruz Biotechnology), rabbit polyclonal anti-Bim antibody (1:1000; Santa Cruz Biotechnology), and mouse monoclonal anti-Actin antibody (1:100,000; Millipore), After several washes in PBS-T, the membranes were incubated with peroxidase-conjugated goat anti-mouse or rabbit IgG (1:5000; Bio-Rad), and developed using enhanced chemiluminescence substrate system. The images were captured and quantified by using ImageQuant LAS 4000 system (GE Healthcare Bio-Science) and the band densities were normalized to the band densities for actin.

\section{SBEM}

$\mathrm{ONH}$ tissues were washed with buffer and then placed into $2 \% \mathrm{OsO}_{4} / 1.5 \%$ potassium ferrocyanide in either 0.15 $\mathrm{M} \mathrm{CB}$ containing $2 \mathrm{mM} \mathrm{CaCl}_{2}$. The tissues were left for 2 $\mathrm{h}$ at room temperature. After thorough washing in double distilled water, the tissues were placed into $0.05 \%$ thiocarbohydrazide for $30 \mathrm{~min}$. The slices were again washed and then stained with $2 \%$ aqueous $\mathrm{OsO}_{4}$ for $1 \mathrm{~h}$. The tissues were washed and then placed into $2 \%$ aqueous uranyl acetate overnight at $4{ }^{\circ} \mathrm{C}$. The tissues were washed with water at room temperature and then stained with en bloc lead aspartate for $30 \mathrm{~min}$ at $60^{\circ} \mathrm{C}$. The tissues were washed with water and then dehydrated on ice in $50,70,90,100,100 \%$ ethanol solutions for $10 \mathrm{~min}$ at each step. The tissues were then washed twice in dry acetone and then placed into 50:50 Durcupan ACM: acetone overnight. The tissues were transferred to $100 \%$ Durcupan resin overnight. The tissues were then embedded and left in an oven at $60{ }^{\circ} \mathrm{C}$ for $72 \mathrm{~h}$. SBEM data were collected with a 3 View unit (Gatan Inc.) installed on a Merlin field emission SEM (Carl Zeiss Microscopy). The ONH volumes were collected in 2.0-2. $4 \mathrm{kV}$ accelerating voltages, with a raster size of $24 \mathrm{k} \times 24 \mathrm{k}$ and pixel dwell time of $0.5-1 \mu \mathrm{s}$. The pixel sizes were $4.0-7.3 \mathrm{~nm}$, depending on the raster size and section thickness was $60-70 \mathrm{~nm}$. Once a volume was collected, the histograms for the tissues throughout the volume stack were normalized to correct for drift in image intensity during acquisition. Digital micrograph files (.dm4) were normalized using Digital Micrograph and then converted to MRC format. The stacks were converted to eight bit and volumes were manually traced for reconstruction and analysis using IMOD software (http://bio3d.colorado.edu/imod//60 .

\section{In vitro transduction of recombinant AAV2/5 constructs}

Recombinant AAV2/5 was prepared by using AAV Helper-Free System (Agilent Technology) with some modification. To generate an AAV2/5 capable of expressing mCherry-PKI fusion protein in astrocytes, AAV2/5 rep, and cap plasmid was purchased from Penn Vector
Core (University of Pennsylvania) and pZac2.1 gfaABC1D-tdTomato ${ }^{61}$ was a gift from Baljit Khakh (Addgene plasmid \# 44332). To construct pZac2.1 gfaABC1D-mCherry-PKI, the cDNA for mCherry-PKI was amplified from p4mt-mCherry-PKI that was kindly provided by Dr. Giulietta Di Benedetto ${ }^{62}$ by using following primers; Forward 5'AAGCTTGAATTC GCCACCATGGTGAGCAAGGGCGAGGAGGA-3' and reverse 5'-AAGCTTGCGGCCGCCTATGACTCGGACT TAGCAG-3'. The tdTomato portion of pZac2.1 gfaABC1D-tdTomato was replaced with mCherry-PKI at EcoR I and Not I site. After DNA sequence validation, these plasmids were used for AAV2/5 production. Viruses were prepared according to manufacturer's instruction (AAV Helper-Free System; Agilent Technology) and purified by a column chromatography using a ViraTrap AAV purification kit (Biomiga) as described in the manufacturer's protocol. Viral titers were determined using a quantitative real-time PCR method. The both AAV2/5 titers were $2.0 \times 10^{12} \mathrm{GC} / \mathrm{ml}$ and transduction was performed to $\mathrm{ONH}$ astrocytes with 400,000 MOI.

\section{Statistical analyses}

Data were presented as the mean \pm S.D. Comparison experimental conditions was evaluated using the twotailed unpaired Student's $t$-test with Bonferroni correction. $p<0.05$ was considered to be statistically significant.

\section{Acknowledgements}

We thank G Di Benedetto for p4mt-mCherry-PKI construct as well as thoughtful comments, and J Lee for thoughtful comments. This work was supported, in part, by NIH grants EY018658 (WKJ), P30EY022589 (Vision Research Core Grant) and 5P41GM103412-28 (MHE), and an unrestricted grant from Research to Prevent Blindness (New York, NY, USA).

\section{Author contributions}

M.S.S., K-Y.K., J.H.B., H.S.N., S.W.J., T.L.P, and W-.K.J. performed experiments and analyzed data. M.S.S., K-Y.K, and W-.K.J designed experiments. M.S.S. and W.-K.J. wrote and revised the manuscript with input from M.H.E and R.N.W.

\section{Competing financial interests}

The authors declare no conflict of interest.

\section{Publisher's note}

Springer Nature remains neutral with regard to jurisdictional claims in published maps and institutional affiliations.

\section{Supplementary information}

The online version of this article (https://doi.org/10.1038/s41419-017-0171-8) contains supplementary material.

Received: 6 September 2017 Revised: 8 November 2017 Accepted: 14 November 2017

Published online: 19 February 2018

\footnotetext{
References

1. Weinreb, R. N. \& Khaw, P. T. Primary open-angle glaucoma. Lancet 363, 1711-1720 (2004)
} 
2. Hernandez, M. R., Miao, H. \& Lukas, T. Astrocytes in glaucomatous optic neuropathy. Prog. Brain Res. 173, 353-373 (2008).

3. Ju, W. K. et al. Increased mitochondrial fission and volume density by blocking glutamate excitotoxicity protect glaucomatous optic nerve head astrocytes. Glia 63, 736-753 (2015).

4. Tezel, G. Oxidative stress in glaucomatous neurodegeneration: mechanisms and consequences. Prog. Retin. Eye Res. 25, 490-513 (2006).

5. Sun, D., Lye-Barthel, M., Masland, R. H. \& Jakobs, T. C. Structural remodeling of fibrous astrocytes after axonal injury. J. Neurosci. 30, 14008-14019 (2010).

6. Dai, C. et al. Structural basis of glaucoma: the fortified astrocytes of the optic nerve head are the target of raised intraocular pressure. Glia $\mathbf{6 0}$, 13-28 (2012)

7. Huneycutt, B. S. \& Benveniste, E. N. Regulation of astrocyte cell biology by the cAMP/protein kinase A signaling pathway. Adv. Neuroimmunol. 5, 261-269 (1995).

8. Ladilov, Y. \& Appukuttan, A. Role of soluble adenylyl cyclase in cell death and growth. Biochim. Biophys. Acta 1842, 2646-2655 (2014).

9. Martinez, J. et al. Soluble adenylyl cyclase is necessary and sufficient to overcome the block of axonal growth by myelin-associated factors. J. Neurosci. 34, 9281-9289 (2014).

10. Shim, M. S., Kim, K. Y. \& Ju, W. K. Role of cyclic AMP in the eye with glaucoma. BMB Rep. 50, 60-70 (2017)

11. Lukas, T. J. et al. Susceptibility to glaucoma: differential comparison of the astrocyte transcriptome from glaucomatous African American and Caucasian American donors. Genome Biol. 9, R111 (2008).

12. Chen, L., Lukas, T. J. \& Hernandez, M. R. Hydrostatic pressure-dependent changes in cyclic AMP signaling in optic nerve head astrocytes from Caucasian and African American donors. Mol. Vis. 15, 1664-1672 (2009).

13. Beal, M. F. Aging, energy, and oxidative stress in neurodegenerative diseases. Ann. Neurol. 38, 357-366 (1995).

14. Jenner, P. Oxidative stress as a cause of Parkinson's disease. Acta Neurol. Scand. Suppl. 136, 6-15 (1991).

15. Coyle, J. T. \& Puttfarcken, P. Oxidative stress, glutamate, and neurodegenerative disorders. Science 262, 689-695 (1993).

16. Noh, Y. H. et al. Inhibition of oxidative stress by coenzyme Q10 increases mitochondrial mass and improves bioenergetic function in optic nerve head astrocytes. Cell Death Dis. 4, e820 (2013).

17. Ogura, M., Taniura, H., Nakamichi, N. \& Yoneda, Y. Upregulation of the glutamine transporter through transactivation mediated by CAMP/protein kinase A signals toward exacerbation of vulnerability to oxidative stress in rat neocortical astrocytes. J. Cell Physiol. 212, 375-385 (2007).

18. Dong, C. J., Guo, Y., Agey, P., Wheeler, L. \& Hare, W. A. Alpha2 adrenergic modulation of NMDA receptor function as a major mechanism of RGC protection in experimental glaucoma and retinal excitotoxicity. Invest. Ophthalmol. Vis. Sci. 49, 4515-4522 (2008).

19. Courilleau, D. et al. Identification of a tetrahydroquinoline analog as a pharmacological inhibitor of the CAMP-binding protein Epac. J. Biol. Chem. 287, 44192-44202 (2012)

20. Garwood, C. J. et al. Insulin and IGF1 signalling pathways in human astrocytes in vitro and in vivo; characterisation, subcellular localisation and modulation of the receptors. Mol. brain 8, 51 (2015).

21. John, S. W. et al. Essential iris atrophy, pigment dispersion, and glaucoma in DBA/2J mice. Invest. Ophthalmol. Vis. Sci. 39, 951-962 (1998).

22. Kaempfer, R. RNA sensors: novel regulators of gene expression. EMBO Rep. 4 1043-1047 (2003).

23. Li, X. et al. Determination of dual effects of parathyroid hormone on skeletal gene expression in vivo by microarray and network analysis. J. Biol. Chem. 282, 33086-33097 (2007).

24. Gangoda, L. et al. Cre transgene results in global attenuation of the CAMP/PKA pathway. Cell Death Dis. 3, e365 (2012).

25. Corredor, R. G. et al. Soluble adenylyl cyclase activity is necessary for retinal ganglion cell survival and axon growth. J. Neurosci. 32, 7734-7744 (2012).

26. Cueva Vargas, J. L., Belforte, N. \& Di Polo, A. The glial cell modulator ibudilast attenuates neuroinflammation and enhances retinal ganglion cell viability in glaucoma through protein kinase A signaling. Neurobiol. Dis. 93, 156-171 (2016).

27. Dao, K. K. et al. Epac1 and CAMP-dependent protein kinase holoenzyme have similar CAMP affinity, but their CAMP domains have distinct structural features and cyclic nucleotide recognition. J. Biol. Chem. 281, 21500-21511 (2006).
28. Srivastava, R. K. et al. Involvement of microtubules in the regulation of $\mathrm{BCl} 2$ phosphorylation and apoptosis through cyclic AMP-dependent protein kinase. Mol. Cell Biol. 18, 3509-3517 (1998).

29. Kumar, S. et al. Suppression of soluble adenylyl cyclase protects smooth muscle cells against oxidative stress-induced apoptosis. Apoptosis 19, 1069-1079 (2014)

30. Franke, T. F., Hornik, C. P., Segev, L., Shostak, G. A. \& Sugimoto, C. PI3K/Akt and apoptosis: size matters. Oncogene 22, 8983-8998 (2003).

31. Durand, D., Carniglia, L., Caruso, C. \& Lasaga, M. Reduced CAMP, Akt activation and p65-c-Rel dimerization: mechanisms involved in the protective effects of mGluR3 agonists in cultured astrocytes. PLOS ONE 6, e22235 (2011).

32. Song, G., Ouyang, G. \& Bao, S. The activation of Akt/PKB signaling pathway and cell survival. J. Cell Mol. Med. 9, 59-71 (2005).

33. Romashkova, J. A. \& Makarov, S. S. NF-kappaB is a target of AKT in antiapoptotic PDGF signalling. Nature 401, 86-90 (1999).

34. Cheng, J., Phong, B., Wilson, D. C., Hirsch, R. \& Kane, L. P. Akt fine-tunes NFkappaB-dependent gene expression during T cell activation. J. Biol. Chem. $\mathbf{2 8 6}$ 36076-36085 (2011).

35. Brunet, A. et al. Akt promotes cell survival by phosphorylating and inhibiting a Forkhead transcription factor. Cell 96, 857-868 (1999).

36. Zhang, X., Tang, N., Hadden, T. J. \& Rishi, A. K. Akt FoxO and regulation of apoptosis. Biochim. Biophys. Acta 1813, 1978-1986 (2011)

37. Dijkers, P. F., Medema, R. H., Lammers, J. W., Koenderman, L. \& Coffer, P. J. Expression of the pro-apoptotic $\mathrm{BCl}-2$ family member Bim is regulated by the forkhead transcription factor FKHR-L1. Curr. Biol. 10, 1201-1204 (2000).

38. Bouillet, P. et al. Proapoptotic $\mathrm{BCl}-2$ relative Bim required for certain apoptotic responses, leukocyte homeostasis, and to preclude autoimmunity. Science 286, 1735-1738 (1999)

39. Willis, $\mathrm{S}$. N. et al. Apoptosis initiated when $\mathrm{BH} 3$ ligands engage multiple $\mathrm{BCl}-2$ homologs, not Bax or Bak. Science 315, 856-859 (2007)

40. Tait, S. W. \& Green, D. R. Mitochondria and cell death: outer membrane permeabilization and beyond. Nat. Rev. Mol. Cell Biol. 11, 621-632 (2010).

41. Murray, A. J. Pharmacological PKA inhibition: all may not be what it seems. Sci. Signal. 1, re4 (2008)

42. Dalton, G. D. \& Dewey, W. L. Protein kinase inhibitor peptide (PKI): a family of endogenous neuropeptides that modulate neuronal CAMP-dependent protein kinase function. Neuropeptides 40, 23-34 (2006).

43. Lochner, A. \& Moolman, J. A. The many faces of H89: a review. Cardiovasc Drug Rev. 24, 261-274 (2006)

44. Meyer-Franke, A., Kaplan, M. R., Pfrieger, F. W. \& Barres, B. A. Characterization of the signaling interactions that promote the survival and growth of developing retinal ganglion cells in culture. Neuron 15, 805-819 (1995).

45. Goldberg, J. L. et al. Retinal ganglion cells do not extend axons by default: promotion by neurotrophic signaling and electrical activity. Neuron 33, 689-702 (2002)

46. Kokotas, H. et al. Biomarkers in primary open angle glaucoma. Clin. Chem. Lab. Med. 50, 2107-2119 (2012)

47. Bhattacharya, S. K., Lee, R. K. \& Grus, F. H., Seventh APORICWG. Molecula biomarkers in glaucoma. Invest. Ophthalmol. Vis. Sci. 54, 121-131 (2013).

48. Yan, X., Tezel, G., Wax, M. B. \& Edward, D. P. Matrix metalloproteinases and tumor necrosis factor alpha in glaucomatous optic nerve head. Arch. Ophthalmol. 118, 666-673 (2000)

49. Yuan, L. \& Neufeld, A. H. Tumor necrosis factor-alpha: a potentially neurodestructive cytokine produced by glia in the human glaucomatous optic nerve head. Glia 32, 42-50 (2000)

50. Tezel, G. TNF-alpha signaling in glaucomatous neurodegeneration. Prog. Brain Res. 173, 409-421 (2008)

51. Tezel, G. \& Wax, M. B. Increased production of tumor necrosis factor-alpha by glial cells exposed to simulated ischemia or elevated hydrostatic pressure induces apoptosis in cocultured retinal ganglion cells. J. Neurosci. 20 8693-8700 (2000)

52. Dringen, R., Pawlowski, P. G. \& Hirrlinger, J. Peroxide detoxification by brain cells. J. Neurosci. Res. 79, 157-165 (2005).

53. Fernandez-Fernandez, S., Almeida, A. \& Bolanos, J. P. Antioxidant and bioenergetic coupling between neurons and astrocytes. Biochem. J. 443, 3-11 (2012).

54. Davila, D. Fernandez, S. \& Torres-Aleman, I. Astrocyte resilience to oxidative stress induced by insulin-like growth factor I (IGF-I) involves preserved AKT (Protein Kinase B) activity. J. Biol. Chem. 291, 2510-2523 (2016).

55. Mackey, A. M. et al. Redox survival signalling in retina-derived $661 \mathrm{~W}$ cells. Cell Death Differ. 15, 1291-1303 (2008). 
56. Eftekharzadeh, B. et al. Inhibition of PKA attenuates memory deficits induced by beta-amyloid (1-42), and decreases oxidative stress and NF-kappaB transcription factors. Behav. Brain Res. 226, 301-308 (2012).

57. Yan, L. et al. Type 5 adenylyl cyclase disruption increases longevity and protects against stress. Cell 130, 247-258 (2007).

58. Kim, K. Y. et al. DRP1 inhibition rescues retinal ganglion cells and their axons by preserving mitochondrial integrity in a mouse model of glaucoma. Cell Death Dis. 6, e1839 (2015).

59. Barres, B. A., Silverstein, B. E., Corey, D. P. \& Chun, L. L. Immunological, morphological, and electrophysiological variation among retinal ganglion cells purified by panning. Neuron 1, 791-803 (1988)
60. Kremer, J. R., Mastronarde, D. N. \& Mclntosh, J. R. Computer visualization of three-dimensional image data using IMOD. J. Struct. Biol. 116, 71-76 (1996).

61. Shigetomi, E. et al. Imaging calcium microdomains within entire astrocyte territories and endfeet with GCaMPs expressed using adeno-associated viruses. J. Gen. Physiol. 141, 633-647 (2013).

62. Di Benedetto, G., Scalzotto, E., Mongillo, M. \& Pozzan, T. Mitochondrial Ca(2)(+) uptake induces cyclic AMP generation in the matrix and modulates organelle ATP levels. Cell Metab. 17, 965-975 (2013). 